\title{
Strength of Integration of Transmembrane $\alpha$-Helical Peptides in Lipid Bilayers As Determined by Atomic Force Spectroscopy ${ }^{\dagger}$
}

\author{
Dragomir N. Ganchev, \\ Department of Biochemistry of Membranes, Faculty of Chemistry, Institute of Biomembranes, Department of Condensed Matter \\ and Interfaces, Faculty of Chemistry, and Department of Medicinal Chemistry, Faculty of Pharmaceutical Sciences, \\ Utrecht University, Utrecht, The Netherlands
}

Received July 30, 2004; Revised Manuscript Received September 17, 2004

\begin{abstract}
In this study we address the stability of integration of proteins in membranes. Using dynamic atomic force spectroscopy, we measured the strength of incorporation of peptides in lipid bilayers. The peptides model the transmembrane parts of $\alpha$-helical proteins and were studied in both ordered peptiderich and unordered peptide-poor bilayers. Using gold-coated AFM tips and thiolated peptides, we were able to observe force events which are related to the removal of single peptide molecules out of the bilayer. The data demonstrate that the peptides are very stably integrated into the bilayer and that single barriers within the investigated region of loading rates resist their removal. The distance between the ground state and the barrier for peptide removal was found to be $0.75 \pm 0.15 \mathrm{~nm}$ in different systems. This distance falls within the thickness of the interfacial layer of the bilayer. We conclude that the bilayer interface region plays an important role in stably anchoring transmembrane proteins into membranes.
\end{abstract}

One out of three cellular proteins is a membrane protein because its sequence contains one or more stretches of hydrophobic amino acids that span the membrane as an $\alpha$-helix. This motive allows for energetically favorable hydrogen bonding of the peptide backbone within the hydrophobic interior of the membrane. The hydrophobic helix in combination with flanking aromatic amino acids such as tryptophan ensures stable integration of intrinsic proteins into the lipid bilayer $(1,2)$. The bilayer is a complex and dynamic structure consisting of a hydrocarbon layer that is separated from the aqueous medium by a broad, water-rich interfacial region consisting of the headgroups, the glycerols, and ester bonds (1).

Estimates on the membrane affinity of the hydrophobic helix are available (1). But precisely how stable are proteins integrated into membranes and what is the determinating factor for their stable integration? These are unanswered and fundamental questions in membrane biology that we address in this study.

To get insight into these questions, a range of techniques such as optical tweezers (3) and biomembrane force probes (4) are available. Among these, atomic force microscopy $(\mathrm{AFM})^{1}$ is unique because it can combine spatial information with force measurements. Ideally, one would like to measure the strength of integration of a membrane protein in its

\footnotetext{
$\dagger$ This study was financially supported by a grant from the NWOCW.

* Correspondence should be directed to this author. Tel: +31302531607. Fax: +31302533969. E-mail: b.dekruijff@chem.uu.nl

Department of Biochemistry of Membranes, Faculty of Chemistry, Institute of Biomembranes, Utrecht University.

$\S$ Department of Condensed Matter and Interfaces, Faculty of Chemistry, Utrecht University.

"Department of Medicinal Chemistry, Faculty of Pharmaceutical Sciences, Utrecht University.
}

natural surroundings. This has been done for bacteriorhodopsin and has resulted in insight into the unfolding pathway of this protein (5). However, interpretation of such studies in terms of contributions of specific parts of the protein and its relation to its surrounding is hampered by the complexity of such systems. Therefore, in this study we analyzed the strength of integration of transmembrane peptides consisting of a single $\alpha$-helix that spans the bilayer. Such peptides can be designed to model the transmembrane parts of proteins and are commonly used in membrane research (6).

The peptides used here are well characterized $(6-8)$ and consist of a simple stretch of hydrophobic amino acids (alanine-leucine) of defined length, flanked on either side by two tryptophans. These peptides incorporate as transmembrane $\alpha$-helices into bilayers. We functionalized these peptides on their $\mathrm{N}$-terminus with a thio group and incorporated them into supported bilayers of phosphatidylcholine, an abundant natural membrane lipid. We studied peptides dispersed in fluid bilayers as well as peptides organized in highly organized peptide-rich domains (8). In these domains the peptides interact with each other and the surrounding lipids, thus mimicking multispan membrane proteins. We probed the strength of integration of the peptides into the bilayers by pulling at them with a gold-covered AFM tip, and we observed distinct events corresponding to the removal of single peptides from the bilayer.

The results provide for the first time information on the strength of integration of $\alpha$-helical peptides incorporated into lipid bilayers and lead to the conclusion that the bilayer

${ }^{1}$ Abbreviations: DPPC, 1,2-dipalmitoyl-sn-glycero-3-phosphocholine; AFM, atomic force microscopy; Ac-WALP, acetylated GWW(LA) ${ }_{8} \mathrm{LWWA}-\mathrm{NH}_{2}$ peptide; SH-WALP, thioglycolic acid-GWW$(\mathrm{LA})_{8} \mathrm{LWWA}-\mathrm{NH}_{2}$ peptide. 
interface region plays an important role in stable integration of transmembrane $\alpha$-helices in membranes.

\section{EXPERIMENTAL PROCEDURES}

Materials. 1,2-Dipalmitoyl-sn-glycero-3-phosphocholine (DPPC) and 1,2-dioleoyl-sn-glycero-3-phosphocholine (DOPC) were obtained from Avanti Polar Lipids (Alabaster, AL).

Peptides were prepared by solid-phase synthesis and purified and handled as described (8) with some modifications as will be described elsewhere to introduce an $\mathrm{N}$ terminal free $\mathrm{SH}$ group. The peptides used in this study were [SH-CH 2 -CO]GWWL(AL) $)_{8}$ WWA-NH ${ }_{2}$ (SH-WALP23), $\left[\mathrm{CH}_{3}-\right.$ COJGWWL(AL) ${ }_{8}$ WWA-NH ${ }_{2}$ (Ac-WALP23), and CWWL(AL) ${ }_{10} \mathrm{WWA}-\mathrm{NH}_{2}$ (Cys-WALP27). The purity of the peptides was $\geq 95 \%$. Their identity was confirmed by electrospray ionization mass spectrometry.

Preparation of Supported Bilayers. Peptide-containing supported bilayers on mica were prepared via the vesicle fusion method as described in ref 8 . Small unilamellar vesicles (SUV) were prepared by hydrating a mixed peptide/ lipid film of desired composition with a $20 \mathrm{mM} \mathrm{NaCl}$ solution (1 mM lipid), containing $0.05 \mathrm{mM}$ dithiothreitol (DTT), followed by 10 freeze-thaw cycles and sonication in a bath sonicator at maximum power for $30 \mathrm{~min}$ at $45^{\circ} \mathrm{C}$, followed by a $1 \mathrm{~h}, 20800 \mathrm{~g}$ spin at $4{ }^{\circ} \mathrm{C}$. The SUV-containing supernatant $(75 \mu \mathrm{L})$ was deposited onto freshly cleaved mica, followed by a $1 \mathrm{~h}$ incubation at room temperature to allow absorption of the vesicles. Subsequently, the sample was rinsed three times with $75 \mu \mathrm{L}$ of the $\mathrm{NaCl} / \mathrm{DTT}$ solution, heated for $1 \mathrm{~h}$ at $65^{\circ} \mathrm{C}$, cooled to room temperature, and washed again three times with the solution. The resulting supported bilayer was covered with the solution during the AFM measurements.

AFM Imaging. The samples were mounted on an Escanner, which was calibrated on a standard grid of a nanoscope III AFM (Digital Instrument, Santa Barbara, CA). A fluid cell without O-ring was fitted, and the sample was scanned in contact mode, using either oxide-sharpened $\mathrm{Si}_{3} \mathrm{~N}_{4}$ tips attached to a triangular cantilever with a spring constant of $0.06 \mathrm{~N} / \mathrm{m}$ (Nano Probe Digital Instruments) or goldcovered tips mounted on a rectangular cantilever with a spring constant of 0.027 or $0.006 \mathrm{~N} / \mathrm{m}$ (Olympus Bio-Lever BL-RC $150 \mathrm{VB})$. Images were recorded at a minimal force $(100-200 \mathrm{pN})$ where the scans were stable and at temperatures between 23 and $28{ }^{\circ} \mathrm{C}$.

AFM Force Measurements. After visualization of the image with a tip speed of $10 \mu \mathrm{m} / \mathrm{s}$, the gold-coated tips were positioned on the spot of interest whereafter force-distance curves were recorded in the retraction cycle. By varying the vertical velocity of the scanner and by using gold-coated cantilevers of different spring constants, different loading rates were applied. These tips were calibrated using the Hutter method (9), yielding values of the spring constant of 0.025 and $0.007 \mathrm{~N} / \mathrm{m}$, which was close to the values quoted for these tips of 0.027 and $0.006 \mathrm{~N} / \mathrm{m}$, respectively. The maximum force exerted by the tip (with a measured spring constant of $0.025 \mathrm{~N} / \mathrm{m}$ ) on the bilayer was lower than $1 \mathrm{nN}$, and in the case of the softer cantilever this force was below $400 \mathrm{pN}$. Data sets were obtained with different tips, but in each case at least two loading rates were investigated per tip.

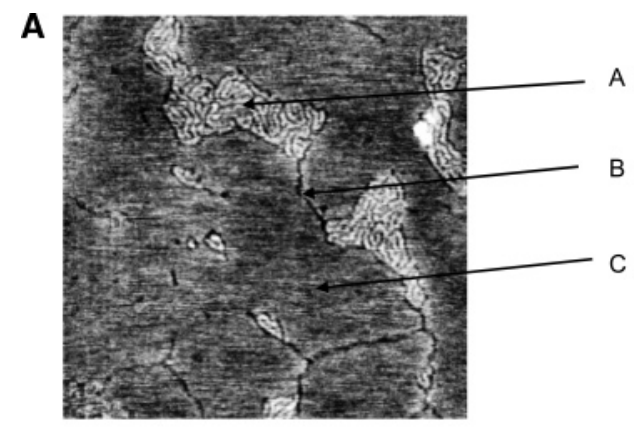

B

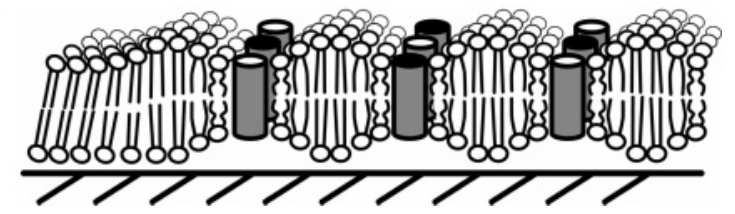

FIGURE 1: (A) AFM image of striated domains in $2 \mathrm{~mol} \% \mathrm{SH}-$ WALP23-containing DPPC bilayers, showing striated domains (arrow A), cracks (arrow B), and the flat bilayer surface (arrow C). Image size is $500 \times 500 \mathrm{~nm}$, and Z-scale is $3 \mathrm{~nm}$. (B) Schematic illustration of the molecular organization of striated domains. See text for further details.

From the statistical distribution of the unbinding events (at least one hundred measured per condition) the most probable unbinding force was plotted versus the natural logaritm of the loading rate. In this analysis only forcedistance curves were included in which the nominal loading rate as determined from the slope of the curve prior to the rupture (10) was within $20 \%$ of the loading rate applied.

Because of the inherent uncertainty in determining the cantilever spring constant $(\sim 20 \%)$, the points obtained with different AFM tips were normalized on a single curve, and the slope of this curve was determined. Then the $X$-intercept was determined from the best of the experimental points.

\section{RESULTS}

Ordered Peptide-Rich Domains as a System for Force Spectroscopy. Transmembrane peptides with aromatic flanking residues and a core of alternating leucines and alanines spontaneously form highly ordered domains with lipids when incorporated in gel state dipalmitoylphosphatidylcholine (DPPC) bilayers and inspected by AFM (8). This is also the case for the peptides analyzed in this study. Figure 1A shows as an example an AFM image of the SH-WALP peptide incorporated at $2 \mathrm{~mol} \%$ in DPPC and scanned with a conventional AFM tip. The image shows the smooth surface corresponding to gel state DPPC and domains that consist of regularly spaced dark lines (repeat distance of $8 \pm 0.5$ $\mathrm{nm}$ ) separated by lighter areas. The domains are typically connected by cracks (dark lines). Scanning the system with a gold-coated tip yielded similar images (Figure 2). The image visualizes a supported bilayer in which line-type depressions and striated domains are present. The black hole at the bottom of the image is a defect in the bilayer (hole down to the mica), through which we could confirm the presence of the intact supported bilayer with a thickness of $\sim 6 \mathrm{~nm}$. The resolving power of the gold-covered tip is less than that of the $\mathrm{Si}_{3} \mathrm{~N}_{4}$ tip, most likely because it is more blunt.

The molecular organization of these so-called striated domains (8; E. Sparr, D. N. Ganchev, J. A. Killian, and B. 


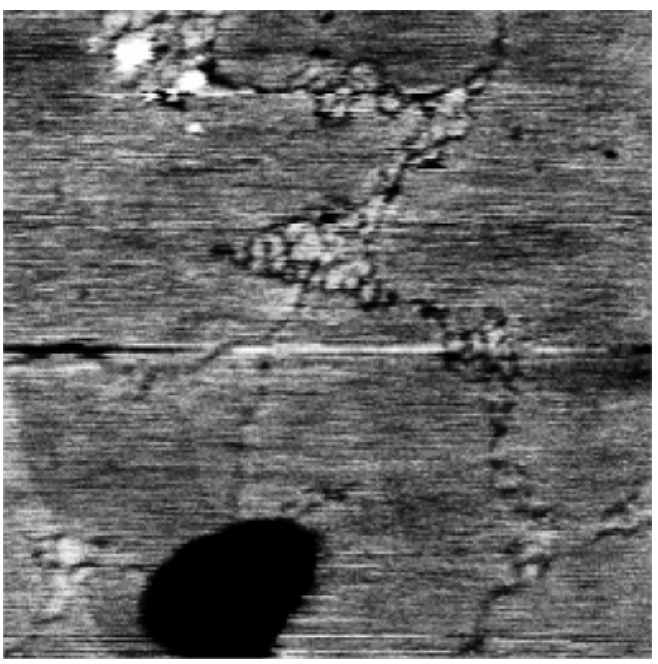

FIGURE 2: AFM image of striated domains in $2 \mathrm{~mol} \% \mathrm{SH}-$ WALP23-containing DPPC bilayers, visualized with a gold-covered tip.

De Kruijff, in press) is illustrated in Figure 1B. The domains consist of regularly spaced linear assemblies of antiparallel transmembrane peptides. In these assemblies the peptides interact with each other and with the surrounding lipids which are disordered because of the peptide-lipid interaction. The lipids in between the rows of peptides have lost the tilt that the acyl chains of DPPC have outside the domains. The organization of the domains and its lipid-protein ratio of 8 are independent of the protein concentration. The striated domains of the thiopeptide can be specifically decorated with $8 \mathrm{~nm}$ sized gold particles (not shown), demonstrating that the $\mathrm{SH}$ group is accessible to a gold surface approaching the bilayer.

We consider the striated domains to be a very useful system for peptide-pulling experiments because the localization of the peptide is known and its surroundings mimic the situation encountered for $\alpha$-helices in complex multispan proteins where also both peptide-peptide and peptide-lipid contacts occur.

Peptide Pulling in Striated Domains. When the goldcovered AFM tip is positioned onto the striated domains of a 2 mol \% SH-WALP23-containing bilayer, upon pulling in more than $75 \%$ of the cases one or more negative peaks preceding the horizontal line in the force-distance $(f-d)$ curves are observed, demonstrating tip-sample interactions (11). In about $70 \%$ of the cases one peak is observed such as shown for curves $2 \mathrm{a}-\mathrm{c}$ in Figure 3. In $25 \%$ of the cases two peaks are observed (curve 3$)$, and in rare cases $(\sim 5 \%$ incidence) three or more peaks are observed (curve 4).

When the gold-covered AFM tip is positioned onto the bilayer but outside the striated domains and retracted, forcedistance $(f-d)$ curves as shown in Figure 3 (curve 1) are observed. These curves, which were observed in more than $99 \%$ of the cases, are typical for a tip that does not interact with the sample.

The tip-sample interactions observed are specific because (i) when the tip is placed over domains formed by the thiofree control peptide Ac-WALP23, in less than 5\% of the cases an interaction is observed, and (ii) when a conventional (non-gold-coated) tip is placed over the domains, in less than $1 \%$ of the cases an interaction is observed.

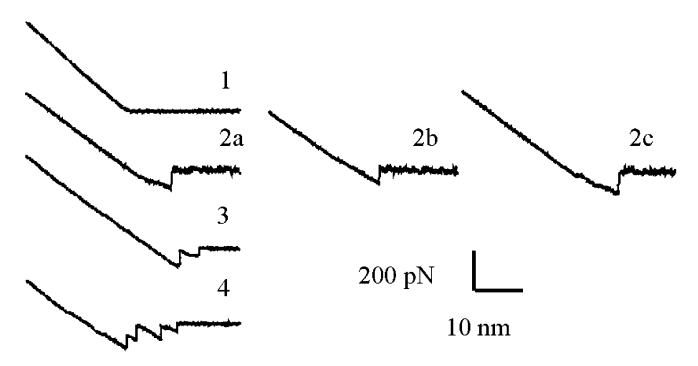

FIGURE 3: Representative retraction force-distance curves recorded on different areas of a 2 mol \% SH-WALP23-containing DPPC bilayer using a gold-covered AFM tip. Curve 1 is representive for the peptide-free DPPC domains whereas curves $2-4$ are obtained on the striated domains.

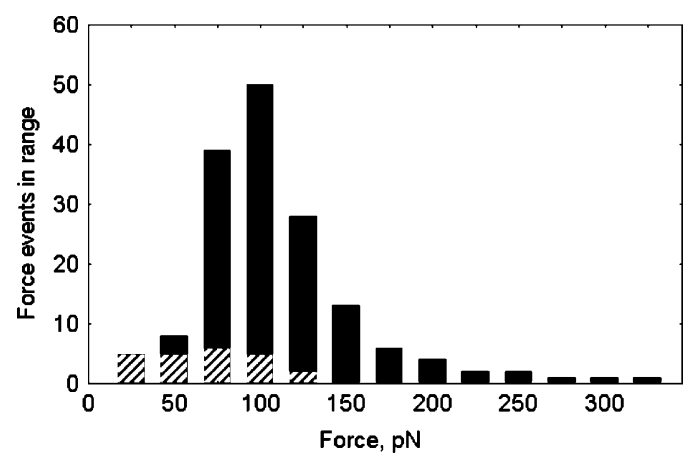

FIGURE 4: Distribution of force events registered with a goldcovered AFM tip over striated domains present in $2 \mathrm{~mol} \% \mathrm{SH}-$ WALP23 (solid bars) or Ac-WALP23 (striped bars) containing DPPC bilayers. A loading rate of $45 \mathrm{nN} / \mathrm{s}$ was used. In $f-d$ curves with more than one peak, only the last peak was evaluated because it reflects the strength of the last bond between the tip and the sample.

A histogram of the distribution of registered force for SHWALP23 over striated domains is shown in Figure 4. For the SH-WALP23 a pronounced peak around $100 \mathrm{pN}$ is observed in the force distribution whereas for the control the much less numerous force events did not show a pronounced peak. The data strongly indicate that upon pulling with the gold-coated tips we extract thiopeptides from the bilayer. It is very unlikely that we measure the breaking of the gold - thio bond because under these conditions this bond has a much higher rupture strength of $1.4 \mathrm{nN}$ (12).

How can we derive quantitative information from the pulling experiments? Breaking noncovalent bonds between biomolecules depends on the rate at which the external force is applied $(13,14)$. In the AFM pulling experiment we load the system at a certain rate. This loading rate $r$ (in $\mathrm{pN} / \mathrm{s}$ ) is the speed by which the cantilever moves away from the sample multiplied by the spring constant of the cantilever. In this case (13) the most probable force measured, $F$, depends on $r$ according to

$$
F=\left(k T / X_{\mathrm{b}}\right) \ln \left(r /\left(k T / X_{\mathrm{b}}\right) K_{\text {off }}\right)
$$

in which $X_{\mathrm{b}}$ is the distance between the ground and transition state, $k T$ is the thermal energy, and $K_{\text {off }}$ is the natural offrate of the complex.

By analyzing the relation between the force events measured and the loading rate, we obtain information on the occurrence of the energy barriers and the thermal off-rates at these barriers. 


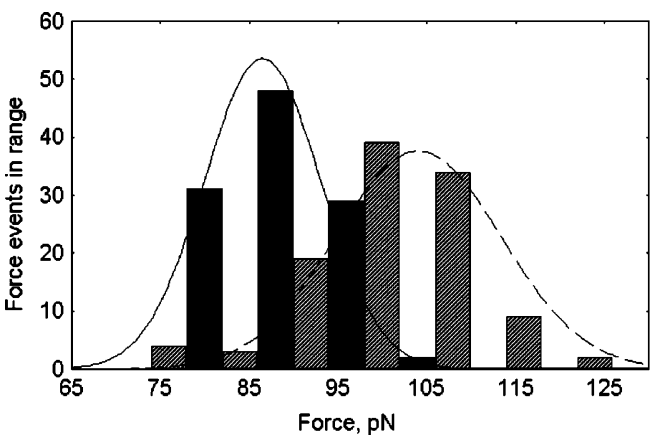

FIGURE 5: Distribution of force events registered on striated domains present in $2 \mathrm{~mol} \%$ SH-WALP-containing DPPC bilayers at loading rates of $4.8 \mathrm{nN} / \mathrm{s}$ (solid bars) and $45 \mathrm{nN} / \mathrm{s}$ (striped bars). The curves are Gaussian fits of the data.

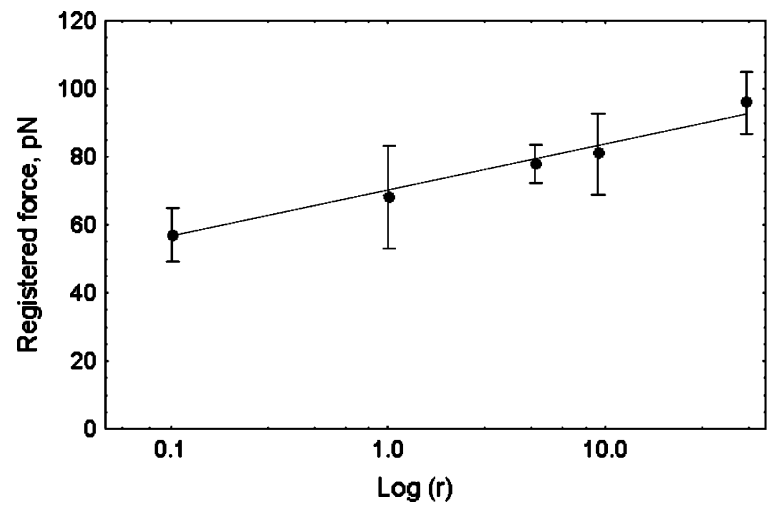

FIGURE 6: Loading rate dependency of the mean force events registered over striated domains present in 2 mol \% SH-WALP23containing DPPC bilayers. Each point is the value and its standard deviation obtained from the Gaussian fit of the distribution of force events recorded.

We probed the strength of the registered interaction of the gold-coated tips with SH-WALP23 domains at loading rates ranging from 0.1 to $45 \mathrm{nN} / \mathrm{s}$. As examples, histograms for the measured force distribution at two different loading rates are shown in Figure 5. The Gaussian fits of the data points yield values of $78 \pm 6$ and $96 \pm 9 \mathrm{pN}$ for loading rates of 4.8 and $45 \mathrm{nN} / \mathrm{s}$, respectively, and demonstrate that the unbinding force increases with an increase in loading rate as expected from theory. The average unbinding forces plotted as a function of the logaritm of the loading rate yield a straight line, which indicates a single barrier for peptide extraction (Figure 6). From the slope the position of this barrier relative to the ground state can be calculated to be $X_{\mathrm{b}}=0.75 \pm 0.15 \mathrm{~nm}$. From the $X$-intercept a $K_{\text {off }}$ of $10^{-4}$ $10^{-5} \mathrm{~s}^{-1}$ can be estimated. Pulling experiments on striated domains of 2 mol \% containing Cys-WALP27, which has a four amino acid longer membrane spanning domain, gave qualitatively similar results (data not shown). Interestingly, for this peptide a lower value of $X_{\mathrm{b}}=0.5 \pm 0.1 \mathrm{~nm}$ and a $K_{\text {off }}$ of $10^{-4} \mathrm{~s}^{-1}$ were measured.

These data demonstrate that quantitative information on the strength of integration of a peptide in the ordered peptiderich domains can be determined by atomic force spectroscopy.

Peptide Pulling in Fluid Bilayers. Many proteins span the membrane with single hydrophobic $\alpha$-helices that are embedded in and interact with the fluid bilayer.

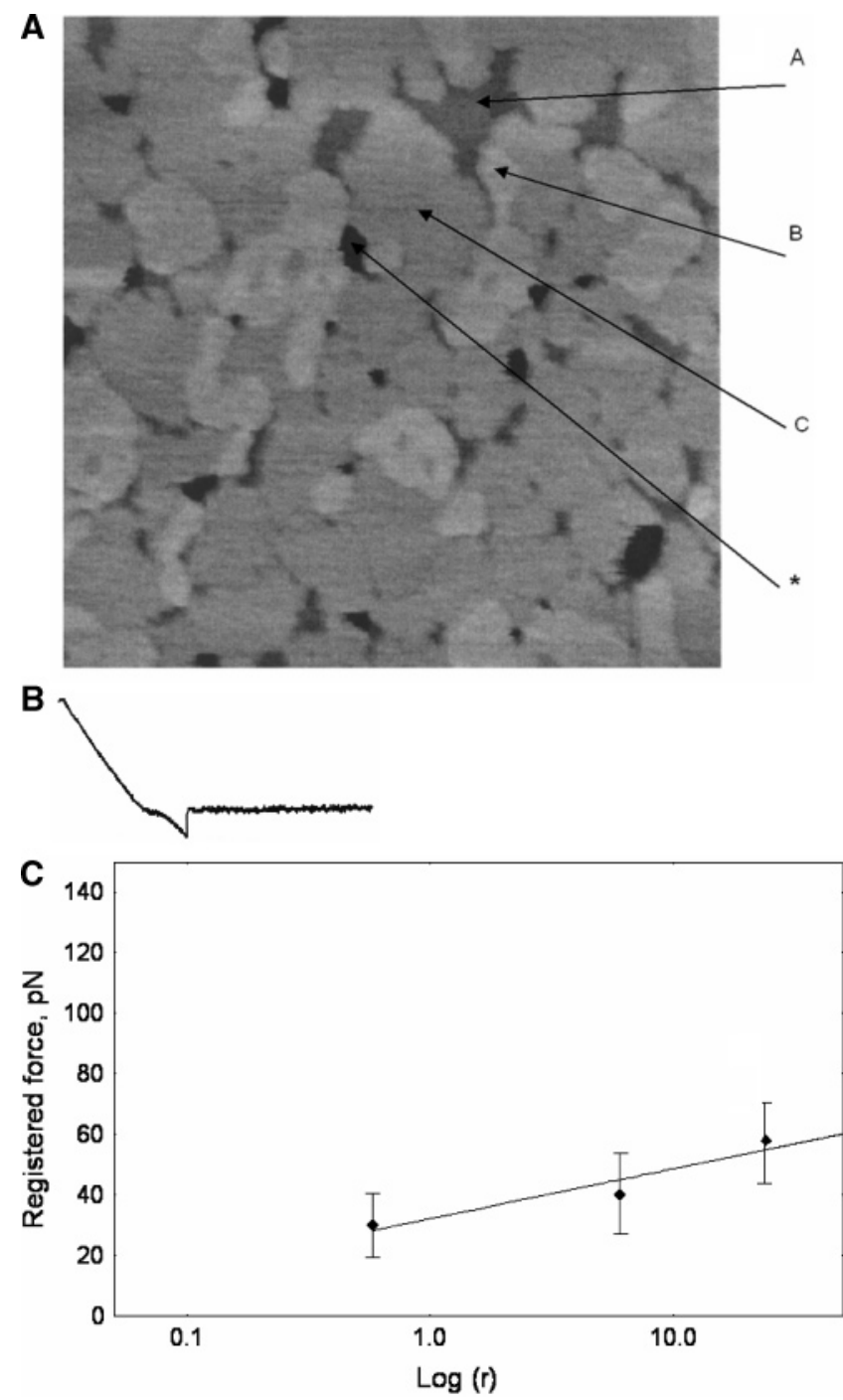

FIGURE 7: Peptide extraction from fluid DOPC domains. (A) AFM image (size $500 \times 500 \mathrm{~nm}, Z$-scale $15 \mathrm{~nm}$ ) of a supported bilayer of $0.5 \mathrm{~mol} \% \mathrm{SH}-\mathrm{WALP} 23$ in DOPC/DPPC (1:9) showing bilayer domains of DOPC (arrow A) and DPPC (arrow B) or domains with a monolayer of DOPC and DPPC (arrow C). The asterisk indicates a defect in the bilayer down to the mica. (B) Typical force event recorded during retraction of the gold-covered tip from a DOPC bilayer domain. In rare cases multiple events such as shown in curves 3 and 4 of Figure 3 were observed. (C) Loading rate dependency of the mean force events registered on DOPC bilayer domains. Each point is the value and its standard deviation of the Gaussian fit of the distribution of force events recovered. The data were obtained with a single AFM tip.

To model such a situation, we can incorporate $\mathrm{SH}-$ WALP23 in DOPC that forms fluid bilayers at room temperature. In this system the peptide is homogeneously distributed as individual transmembrane $\alpha$-helices (6).

To confine the peptide in an area that can be localized by AFM, we created fluid bilayer domains of DOPC within supported bilayers of gel state DPPC. An AFM image of such a system is shown in Figure 7A. The dark (low) areas (see arrow A) correspond to DOPC bilayer domains that are surrounded by either areas of DPPC bilayers (arrow B) that are $1 \mathrm{~nm}$ higher (lighter) due to acyl chain ordering or areas of bilayers with intermediate darkness that consist of a monolayer of DOPC and a monolayer of DPPC (arrow C).

In such systems the SH-WALP23 peptide is expected to be localized in the fluid DOPC (15). Indeed, when the gold- 
covered AFM tip is positioned on such a DOPC domain, upon pulling force-distance curves typical of an interaction between the tip and the sample are observed (Figure 7B). Analysis of force-distance curves of this system at different loading rates (Figure 7C) revealed a value of $X_{\mathrm{b}}$ of $0.7 \mathrm{~nm}$ and a value for $K_{\text {off }}$ of $6 \times 10^{-4}$. These values are strikingly similar to these observed for the peptide in the very different striated domains and suggest that removal of a transmembrane helix from a membrane occurs through a common mechanism.

\section{DISCUSSION}

In our pulling experiments on transmembrane $\alpha$-helical peptides in different bilayers we observed specific force events. We first discuss the nature of these events. It is unlikely that they relate to lifting of the bilayer or creating a lipid tether (16) because in these cases the retract part of the force-distance curves after the last peak would not coincide with the approach part of the curve, as we observed. Furthermore, the striated domains with their high content of peptide and embedding into gel state DPPC bilayers are expected to strongly resist lifting or bending of the supported bilayer. Finally, the very similar behavior observed for the ordered and disordered bilayer systems also strongly argues against these possibilities. We therefore conclude that the events we observe in the pulling experiments reflect the removal of the peptide out of the bilayer. Given the large number of cases in which single events were observed, we most often register the removal of single peptides that are attached to the tip. That this is true even for the peptide-rich striated domains can be understood from the alternating outin organization in which two SH group-containing N-termini are separated by approximately $2 \mathrm{~nm}$ (see Figure 1B). In cases of rare multiple force events most likely two or more peptides are simultaneously attached to the tip and removed from the bilayer upon retraction. In theory, it is possible that the C-termini of the peptides are interacting with the mica and that we measure the detachment of the peptide from the mica. We exclude this possibility because the C-terminus of the peptide is uncharged, thereby excluding favorable electrostatic interactions. Moreover, it is known that these peptides are localized with their tryptophans in the ester carbonyl region of the phospholipids (17). Since in the case of SH-WALP there is only a single alanine present Cterminal to these tryptophans, the $\mathrm{C}$-terminus will reside within the phospholipid headgroup region, which is separated by about $1 \mathrm{~nm}$ of water from the mica. Finally, for bacteriorhodopsin (BR) present in a supported purple membrane it was found that the forces associated with the removal of helices out of the membrane were similar for membranes absorbed onto mica, graphite, or another purple membrane (18). This demonstrates that even the more extended loops in BR connecting the transmembrane helices are not stably interacting with the mica. Therefore, we conclude that the removal of the peptide out of the bilayer is not influenced by the peptide-mica interaction.

It is of interest to compare the most probable unbinding forces of the different WALP-containing systems at loading rates used in the literature on related systems.

For this comparison we selected a loading rate of $45 \mathrm{mN} /$ s. By intra- and extrapolation of our data we obtain comparable, most probable unbinding forces of $90 \mathrm{pN}$ for extraction of both WALP23 and WALP27 out of striated domains, demonstrating that increasing the length of the helix does not significantly affect the force it takes to remove it out of the bilayer. It takes similar values of $100-200 \mathrm{pN}$ to extract the helices of BR out of the membrane $(5,18)$. Given the large differences between these systems (multispan versus single span, different composition of helices, and lipid environment), this suggests that removal of a helix out of a membrane occurs through a common mechanism, not very dependent on the chemistry of the system. For the DOPC domains it takes slightly less force $(60 \mathrm{pN})$ to remove the WALP23 out of the bilayer, which can be understood from the more fluid character of these domains. For comparison, to remove a di-C18:0 lipid out of a stearoyl/oleoyl phosphatidylcholine bilayer requires only $20-40 \mathrm{pN}$ (4), demonstrating that transmembrane helices are much more stably integrated in a bilayer than a diacyl membrane lipid.

From the analysis of the loading rate depending of the force to extract a peptide from the bilayer, we could estimate the thermal off-rate of spontaneous detachment of the peptide from the bilayer. For striated domains $K_{\text {off }}$ values of $10^{-4}$ $10^{-5} \mathrm{~s}^{-1}$ were observed, which corresponds with lifetimes of the peptide in the bilayer in the order of hours or days. For DOPC domains a slightly larger value of $K_{\text {off }}$ was estimated. These values are several orders of magnitude lower than that reported for di-C16:0 lipids (4) and are only an order of magnitude higher than the value reported for the extremely stable biotin-avidin complex (10). This again demonstrates that the strength of integration of a transmembrane peptide is very high and belongs to the most stable noncovalent biological interactions reported so far $(10,19$, 20).

We detected single barriers for removal of the $\alpha$-helices out of the bilayer. For the WALP23 peptide, which has a hydrophobic length that closely matches the hydrophobic thickness of the bilayer, this barrier was determined to be localized $0.75 \pm 0.15 \mathrm{~nm}$ from the ground state in the striated domain. Surprisingly, a very similar value was observed for the peptide present in the DOPC domains. These systems differ very much in architecture. In one case the peptide resides in a highly ordered peptide-rich bilayer in which the peptide interacts both with neighboring peptides and with lipids. In the other case the system is fluid, and the peptide interacts only with the surrounding lipids. This suggests that the value of $0.75 \mathrm{~nm}$ must correspond to a specific and fundamental property of the bilayer. What could this be? One obvious possibility is that it relates to the anchoring function of the tryptophans. The tryptophans are positioned near the boundary between the hydrocarbon and interfacial region of the bilayer and anchor the hydrophobic helix at that position. Although the tryptophans will resist being pulled into the hydrocarbon layer or into the interfacial region (17), this resistance is expected to be relatively weak, because tryptophans flanking a hydrophobic $\alpha$-helix can readily move across a bilayer (2l) and their polarity gives them a possibility to move into the interface. Therefore, the barrier must be a property related to moving the hydrophobic $\alpha$-helix out of the hydrophobic core of the bilayer into the waterrich interfacial region. This interfacial region is a broad and dynamical zone that harbors all polar atoms of the membrane lipids. In the WALP23 system there is no significant 


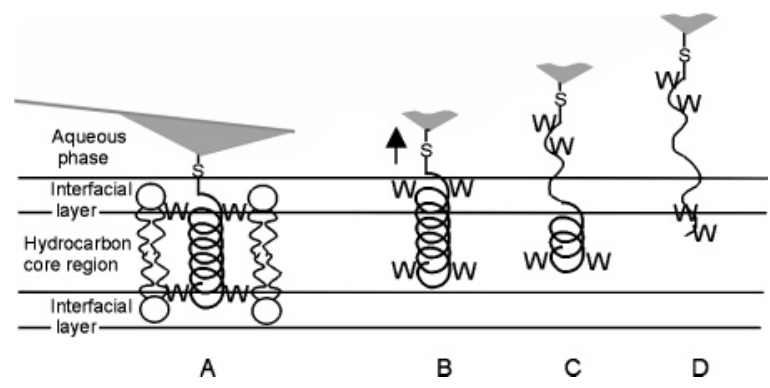

FIGURE 8: Schematic representation of the model describing the mechanical removal of an $\alpha$-helical transmembrane peptide (WALP23) out of the bilayer. The domains of the peptide-lipid interface and hydrocarbon core region are approximately to scale. The gold-coated AFM tip that is chemically attached to the $\mathrm{SH}$ group of the peptide is not to scale. The tryptophans (W) are indicated. The peptide in the ground state is anchored in the bilayer by the tryptophans on both the cis side and the trans side and buries its hydrophobic helix into the hydrocarbon layer (A). When sufficient force is applied to the peptide such that it is displaced $\sim 0.75 \mathrm{~nm}$ from its ground state, it exposes part of the hydrophobic helix to the interface (B). Because of the water present in the interface, the helix will be destabilized and become unfolded upon pulling. At the same time, the displacement of the helix from its ground state results in negative mismatch at the side facing the support, which will make incorporation of the remainder of the peptide in the bilayer less favorable (C). No further major energy barriers are expected upon further pulling, and the peptide is removed from the bilayer (D).

hydrophobic mismatch between the length of the peptide and the thickness of the hydrophobic region of the surrounding fluidized lipid molecules, both being $\sim 3 \mathrm{~nm}$. This implies that pulling on the peptide molecule will expose a growing part of the hydrophobic helix to the water-rich interfacial region. The barrier appears on a distance of $0.75 \mathrm{~nm}$ from the equilibrium position, which corresponds to exposure of four to five residues (a complete turn of the helix) to the aqueous environment. Hydrogen bonding of the peptide backbone to water will destabilize the helix, leading to unfolding and complete withdrawal from the bilayer. This model for the removal of the peptide from the bilayer is visualized in Figure 8 and is further described in its legend.

The model nicely explains our observation that the energy barrier resisting extraction of the longer WALP27 is only $0.5 \mathrm{~nm}$ away from the equilibrium position. The larger peptide has a positive hydrophobic mismatch and therefore already exposes a part of its hydrophobic $\alpha$-helix in the interface (22). The barrier for removal is therefore more readily approached.

The disordered lipids surrounding the peptide are expected to fill the gap in the bilayer caused by removal of the peptide. However, the possibility that some boundary lipids cover the hydrophobic helix upon its extraction, thus protecting it from the aqueous environment, cannot be completely excluded. Yet, this is not very probable, since the removal of the helices of BR out of the purple membrane was proposed to follow a similar helix-unfolding pathway (5) with similar forces needed to extract all helices. In the tightly packed purple membrane there is very small amount of lipids around the BR molecules, and covering all extracted helices with a lipid material seems highly improbable.

In conclusion, we have demonstrated that the hydrophobic $\alpha$-helix of membrane proteins is very stably anchored within the lipid bilayer in a way that is remarkably insensitive to the precise chemical surroundings. It is the bilayer interface that is the barrier resisting mechanical removal of the peptide from the bilayer. This implies that the bilayer interfacial region plays an important role in stable anchoring of proteins in membranes.

\section{ACKNOWLEDGMENT}

The inspiring discussions with Prof. E. Evans, University of British Columbia, are greatly appreciated. The authors are grateful to Kees van der Werf, Twente University, The Nederlands, for assistance with the tip calibrations.

\section{REFERENCES}

1. White, S. H. (2003) Translocons, thermodynamics, and the folding of membrane proteins, FEBS Lett. 555, 116-121.

2. Killian, J. A., and Von Heijne, G. (2000) How proteins adapt to a membrane-water interface, Trends Biochem. Sci. 25, 429-434.

3. Kellermayer, M. S. Z., Smith, S. B., Granzier, H. L., and Bustamante, C. (1997) Folding-unfolding transitions in single titin molecules characterized with laser tweezers, Science 276, 11121116.

4. Evans, E., and Ludwig, F. (2000) Dynamic strengths of molecular anchoring and material cohesion in fluid biomembranes, $J$. Phys.: Condens. Matter 12, A315-A320.

5. Oesterhelt, F., Oesterhelt, D., Pfeiffer, M., Engel, A., Gaub, H. E., and Müller, D. J. (2000) Unfolding pathways of individual bacteriorhodopsins, Science 288, 143-146.

6. De Planque, M. R. R., and Killian, J. A. (2003) Protein-lipid interactions studied with designed transmembrane peptides: role of hydrophobic matching and interfacial anchoring, Mol. Membr. Biol. 20, 271-284.

7. De Planque, M. R. R., Goormaghtigh, E., Greathouse, D. V., Koeppe, R. E., Kruijtzer, J. A. W., Liskamp, R. M. J., De Kruijff, B., and Killian, J. A. (2001) Sensitivity of single membranespanning $\alpha$-helical peptides to hydrophobic mismatch with a lipid bilayer: effects on backbone structure, orientation, and extent of membrane incorporation, Biochemistry 40, 5000-5010.

8. Rinia, H. A., Kik, R. A., Demel, R. A., Snel, M. M. E., Killian, J. A., van der Eerden, J. P. J. M., and De Kruijff, B. (2000) Visualization of highly ordered striated domains induced by transmembrane peptides in supported phosphatidylcholine bilayers, Biochemistry 39, 5852-5858.

9. Hutter, J. L., and Bechhoefer, J. (1993) Calibration of atomicforce microscope tips, Rev. Sci. Instrum. 64, 1868-1873.

10. Dettmann, W., Grandbois, M., Andre, S., Benoit, M., Wehle, A. K., Kaltner, H., Gabius, H.-J., and Gaub, H. E. (2000) Differences in zero-force and force-driven kinetics of ligand dissociation from beta-galactoside-specific proteins (plant and animal lectins, immunoglobulin $\mathrm{G}$ ) monitored by plasmon resonance and dynamic single molecule force microscopy, Arch. Biochem. Biophys. 2, $157-170$.

11. Hoh, J. H., Cleveland, J. P., Prater, C. B., Revel, J.-P., and Hansma, P. K. (1992) Quantitized adhesion detected with the atomic force microscope, J. Am. Chem. Soc. 114, 4917-4918.

12. Grandbois, M., Beyer, M., Rief, M., Clausen-Schaumann, H., and Gaub, H. (1999) How strong is a covalent bond? Science 283 $1727-1730$.

13. Evans E. (1998) Energy landscapes of biomolecular adhesion and receptor anchoring at interfaces explored with dynamic force spectroscopy, Faraday Discuss. 111, 1-16.

14. Evans E., and Ritchie, K. (1997) Dynamic strength of molecular adhesion bonds, Biophys. J. 72, 1541-1555.

15. Van Duyl, B., Rijkers, D. T. S., De Kruijff, B., and Killian J. A. (2002) Influence of hydrophobic mismatch and palmitoylation on the partitioning of transmembrane $\alpha$-helical peptides into detergent resistant domains, FEBS Lett. 523, 79-84.

16. Maeda, N., Senden, T. J., and Di Meglio, J.-M. (2002) Micromanipulation of phospholipid bilayers by atomic force microscopy, Biochim. Biophys. Acta 1564, 165-172.

17. De Planque, M. R. R., Bonev, B. B., Demmers, J. A. A., Greathouse, D. V., Koeppe, R. E., II, Separovic, F., Watts, A., 
and Killian, J. A. (2003) Interfacial anchor properties of tryptophan residues in transmembrane peptides can dominate over hydrophobic mismatch effects in peptide-lipid interactions, Biochemistry $42,5341-5348$.

18. Müller, D. J., Kessler, M., Oesterhelt, F., Möller, C., Oesterhelt, D., and Gaub, H. (2002) Stability of bacteriorhodopsin alphahelices and loops analyzed by single-molecule force spectroscopy, Biophys. J. 83, 3578-3588.

19. Schwesinger, F., Ros, R., Struntz, T., Anselmetti, D., Güntherodt, H.-J., Honegger, A., Jermutus, L., Tiefenauer, L., and Pluckthun, A. (2000) Unbinding forces of single antibody-antigen complexes correlate with their thermal dissociation rates, Proc. Natl. Acad. Sci. U.S.A. 97, 9972-9977.
20. Merkel, R. (2001) Force spectroscopy on single passive biomolecules and single biomolecular bonds, Phys. Rep. 346, 343-385

21. Ridder, A. N. J. A., Morein, S., Stam, J. G., Kuhn, A., De Kruijff, B., and Killian, J. A. (2000) Analysis of the role of interfacial tryptophan residues in controlling the topology of membrane proteins, Biochemistry 39, 6521-6528.

22. Demmers, J. A. A., Van Duijn, E., Haverkamp, J., Greathouse, D. V., Koeppe, R. E., II, Heck, A. J. R., and Killian, J. A. (2001) Interfacial positioning and stability of transmembrane peptides in lipid bilayers as studied by hydrogen/deuterium exchange and mass spectrometry, J. Biol. Chem. 276, 34501-34508.

BI048372Y 